\title{
A Simulation Model and a Hybrid Genetic Algorithm for Energy-Aware MANET Routing and Planning
}

\author{
Ivana Cardial de Miranda Pereira \\ M.Sc \\ Centro de Análise de Sistemas Navais (BrazNavy) \\ COPPE, Federal University of Rio de Janeiro, Brazil
}

\author{
Nelson Francisco Favilla Ebecken \\ D.Sc. \\ COPPE, Federal University of Rio de Janeiro, Brazil
}

\begin{abstract}
This paper presents a new model developed to aid the planning and the analysis of communications-intensive Mobile Ad Hoc Networks (MANET), with respect to the allocation of energy-critical equipment. A graphical simulation tool and a new hybrid genetic algorithm (HGA) are introduced. They work together to estimate the required amount of deployed battery supplies and the probability of success of real operations. At each period, a hybrid genetic algorithm with reparation of individuals and heuristic crossover and mutation operators finds efficient routes that preserve maximum energy availability at network level, reducing the probability of communications disruption. The simulation tool implements mobility models derived from experts' advices and may be used in missions like military and search-and-rescue operations. One may easily include new models to represent the movement of nodes in other specific missions, including trace data. The system is flexible and customizable, providing a means to mission planning, including the provision of adequate power supply for the large number of devices typically included within a MANET.
\end{abstract}

\section{General Terms}

Wireless ad hoc networks.

\section{Keywords}

Genetic Algorithms, Simulation, MANET, Energy Efficiency.

\section{INTRODUCTION}

A mobile ad hoc network (MANET) is a network that has many free or autonomous nodes, composed of mobile devices that can dispose themselves in various ways and operate without strict top-down, administration, or a fixed infrastructure. Each node must forward traffic not necessarily related to its own use, actually acting as a router. The primary challenge in building a MANET is equipping each node to unceasingly keep the information required to correctly route traffic. The development of small smart communications devices, laptops and 802.11/Wi-Fi wireless networking has made MANETs a popular research topic since the mid-1990s. Presently, the ever-growing use of wireless communication technology is raising an import issue: the restrictions imposed by the lifetime of the devices' batteries. MANETs vary from small groups with restricted mobility and low energy requirements, as interactive sessions in universities, to large, highly mobile networks with a dynamic topology and long duration, where energy consumption becomes an important issue.

The design of routing protocols for this kind of network has always been a highly complex task. Due to the unforeseen mobility of the nodes, ad hoc networks present a dynamic topology, i.e., their geometry change frequently and unpredictably, stressing the need for effective and efficient routing protocols. The discovery of viable routes and the efficient delivery of data packets in a decentralized and continuously changing environment is an ill-defined problem. Some relevant technical and topological aspects - inconstant QoS (quality of service), propagation losses, mutual interference, energy consumption, and a highly mobile setup of the entire network - are extremely relevant in this context. Because of that, MANETs must be able to update its routes in an adaptive fashion. In military operations, for example, issues like security, latency, reliability, intentional interference or jamming, and recovery from failure must be seriously considered [1]. These applications typically need coordinated actions, with traffic patterns that obey a rigid hierarchical chain of command, and a "controlled" randomness of the movement of nodes, following a predetermined pattern of mobility, since the groups must pursue, in a collaborative way, common objectives [2].

This work presents new models and computer tools designed to help the planning process of MANETs employed in military and search and rescue operations, or other operations with similar characteristics, like those deployed in inhospitable and/or energy-critical environments. A new hybrid genetic algorithm (HGA) solves the routing problem, aiming to optimize the total energy load of the network, as well as trying to minimize network disruption caused by battery exhaustion by one or more nodes. The main objective here is to increase the probability of mission success. A simulation model to position objectives and moving groups of wireless stations (people, vehicles, etc.) in a limited area of operations is implemented. Beyond producing a graphical presentation of the kinematics of the whole operation, the simulator provides input data for the HGA. Several parameters may be determined to establish the geographical environment scenario, to position stations inside each group, to model each station's mobility on their way to the objective as well as the characteristics of the communication devices, as range and power consumption, etc.

\section{MOBILE AD HOC NETWORK}

\subsection{General Description}

Wireless communications networks may be classified in two principal classes: infrastructured and ad hoc networks. In the first kind, all communications between nodes occur through fixed support stations. Nodes do not communicate directly with each other, even if they are within range.

An ad hoc network, as shown in Figure 1, is composed of a set of independent nodes with wireless communication capability. They communicate directly among themselves, dynamically forming a temporary network, without the need for any central access point or fixed support station. Each 
node, beyond its role as end-user station running applications, works as a router, and is able to discover and maintain a set of possible routes to other nodes.

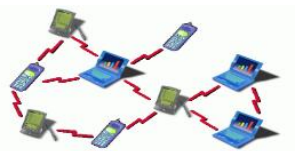

Figure 1 - An ad hoc network

There is no pre-determined topology or a central control station in ad hoc networks, since there is no infrastructure, pre-configured access points or a physical backbone. As a result, these networks can be created very fast, becoming an attractive alternative in situations where setting up an infrastructure is prohibitive because of technological, security or cost issues. The most critical real life applications of a MANET occur in inhospitable environments, or in situations that render the use of an infrastructured network impractical or unsafe.

As examples of ad hoc networks, consider emergency search and rescue operations in places of difficult access; military operations in a limited battlefield; disasters, like earthquakes, hurricanes or flooding; students in interactive classes, conferences, etc. In short, in circumstances where people must disseminate or share information in a short time, using portable devices.

\subsection{Routing}

Ad hoc networks may be divided in direct communication and multi-hop networks. In multi-hop networks, the nodes, beyond being able to communicate with neighbor stations as in direct networks, may reach other stations through multiple "jumps", or hops, when all nodes may operate as routers to receive and forward messages.

Routing consists, basically, in determining an itinerary between two nodes and the actual forwarding of data packets. In order to satisfactorily reach these goals, routing algorithms must fulfil certain operational requirements and determine the best alternative, according to some pre-selected metric. This metric is usually the shortest path, but can also be the least energy consumption, the smallest delay, etc.

In ad hoc networks, due to the constant and unpredictable change in topology caused by the movement of the nodes, routing algorithms must deal robustly with these changes. Furthermore, since the energy available in each station is obviously limited by its battery capacity, it is important to consider the energy consumption at the network level, if one is concerned with the mission this network supports. A large body of research deals with these challenges faced by MANET routing protocols.

\subsection{Scenarios}

The performance of ad hoc networks is one of the main goals of present research concerning MANET routing protocols. However, the great majority of these protocols [3] [4] [5] [6] uses the shortest path as the decision criterion for choosing the best route, neglecting to consider the dynamics of energy consumption. Because of this, some nodes may be selected more frequently than others, increasing the probability of early battery exhaustion [7]. When one node is disabled because of its low battery charge, the protocol routine must recalculate all previously found routes that included that node. One possible serious consequence is the partition of the network if no alternative route exists. In military and search- and-rescue operations, for example, this issue is very important, since reliable communications are critical to mission success. In these scenarios, the shortest path is not necessarily the most adequate metric for choosing the best route. Other mission-related measures must be considered, like energy consumption rate, transmission signal strength, etc.

In a search and rescue operation, different teams of professionals combine efforts to look for survivors in a disaster. Let us suppose that one group is composed of health professionals, including doctors and nurses, and they must be immediately contacted if any victim is found. Another group, comprised of voluntaries, for example, must send the message to the first group, informing the geographical location and the general status of the victim. The immediate change of the network topology as a function of the sudden change in movement of the health service group poses a challenge to the network routing protocol. In addition, it is evident that any interruption of communications in a scenario like this one may lead to dramatic results. In these situations, a routing algorithm must take into account the energy situation of the mobile devices, maximizing the networks service life.

The main characteristic of this real-life scenario, which is explored in this work, is the organization of mobile nodes in cooperative groups, sharing a common objective, but with different mobility patterns. In addition, they have their movements restricted to a limited area of operations and the connection between any pair of nodes depends on their specific task, their device capacity and their relative movement.

\section{GENETIC ALGORITHMS (GA)}

Genetic algorithms simulate natural selection, which sometimes, and not very properly, is known as "survival of the fittest", through mechanisms that emulate the non-random survival of randomly varying hereditary characteristics in a population of coded individuals.

GAs operate on a set of possible solutions. Because of the random nature of GAs, solutions found by an algorithm can be good, poor, or infeasible. To specify how good a solution is, the GA assigns a fitness value to each solution. Chromosomes represent these candidate solutions. The two basic components of chromosomes are the coded solution and its fitness value.

Chromosomes are grouped into a population on which the genetic algorithm operates. At each step of the algorithm, called a generation, the GA selects chromosomes from a population based on their fitness values and combines them to produce new chromosomes (offspring). These offspring chromosomes form a new population, or replace some of the chromosomes in the existing population, in the hope that the new population will be better than the previous ones. The GA keeps track of the best chromosomes and stores additional statistical information that is used to determine its stopping criterion.

A chromosome stores the solution, which it represents using some coding system. There are a number of possible ways to represent a solution so that it is appropriate for the GA (binary, real number, vector of real number, permutations, and so on) and they mostly depend on the nature of the problem.

The basic evolutionary principle of GA's starts with a randomly generated set of individuals represented by chromosomes, called the initial population, and continues with the production of new chromosomes by reproduction. 
This is accomplished by combining existing chromosomes using a crossover operator. A crossover operator takes portions from two existing chromosomes, called parents, and combines them into a new chromosome. Although general crossover operations are easy to implement, building specialized crossover operations for specific problems can greatly improve the performance of the genetic algorithm [8].

After the GA performs a crossover operation, it may execute a mutation operation. A mutation operation makes random, preferably small, changes to an encoded solution, in order to try to prevent the falling of all solutions into a local optimum. This procedure also extends the search space of the algorithm. Mutation and crossover operators depend on the chosen representation of chromosomes. Each individual is evaluated, i.e., the GA computes its fitness, which has the same meaning as the objective function in an optimization problem. The best $N \geq 1$ individuals survive, while others may be eliminated from the new population. The classical structure of a genetic algorithm is shown in Figure 2

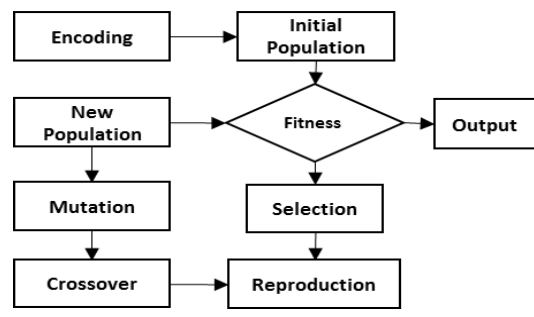

Figure 2 - Classical GA flow chart

To summarize, each iteration of a classical GA corresponds to the application of four basic operations to a population of individuals: fitness evaluation, selection, crossover and mutation. At the end of this sequence of operations, a new population is generated, characterizing the passage of a generation. Hopefully, the new generation represents a better approximation to the best solution of a problem.

\section{ENERGY-AWARE ROUTING}

\subsection{Description}

In this work, it is proposed a new routing algorithm for ad hoc mobile networks supporting real-life operations, based on knowledge about each device's battery charges, so that the network's service life is extended, minimizing the probability of communications disruption, considering communication between two nodes at a time, allowing multiple-hop routes.

\subsection{Modeling the MANET}

A network may be modeled as a set of nodes $\mathrm{N}=\left\{n_{1}, n_{2}, \ldots\right.$, $\left.n_{\mathrm{N}}\right\}$ and a set of links L connecting the nodes in N. A link, or edge, $(i, j)$ exists if the nodes $i$ and $j$ are within communications range of each other. MANETs, then, may be viewed as sequences of graphs $G_{\mathrm{t}}=\left(V_{\mathrm{t}}, E_{\mathrm{t}}\right), t=\{1,2, \ldots, T\}$, where $T$ is the duration of the network, $V_{\mathrm{t}}$ is the set of vertices representing the mobile nodes at period $t$ and $E_{t}$ is the set of possible wireless connections at $t . E_{t}$ is a function of the distance among the nodes and the range of their devices. The number of possible routes from a source to a single destination depends on the size and topology of the network. If the network is large or densely connected, the number of possible routes increase exponentially.

Consider the following small network, shown in Figure 3, with 10 nodes and 17 links. Source and destination nodes are painted gray and receive numbers 1 and 5, respectively. The remaining nodes may play the role of routers, forwarding messages from source to destination.

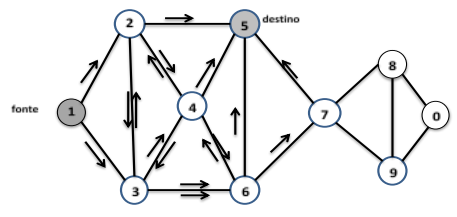

Figure 3 - MANET with 10 nodes

Energy management is carried out at network level and it is assumed that most of the energy is consumed during transmission and reception of data packets. To transmit a packet from node 1 to node 5 , several different routes may be used. The set of possible routes is $\{(1-2-5) ;(1-2-4-5) ;(1-3-2-$ $5) ;(1-3-4-5) ;(1-3-6-5) ;(1-3-4-2-5) ;(1-2-4-6-5) ;(1-2-3-6-4-$ $5) ;(1-3-6-7-5) ;(1-2-3-4-6-7-5) ; \ldots\}$. In a small network like this one, complexity of the search is low, but the search space grows exponentially with the number of the nodes. In addition, in mobile networks routes may frequently vanish due to the movement of the nodes.

\subsection{Proposed Solution}

The computer model is composed of three complementary modules:

- MANET Simulator - it is a multi-hop, mobile wireless network simulation model. It creates an area of operation with a reflecting boundary, a mission objective and deploys operational groups according to one of a set of synthetic or trace mobility models, proposed by specialists and used in missions like military and search-and-rescue operations. One may readily include new models to represent the movement of nodes in other specific missions, including trace data. It shows a graphical representation of the MANET and provides an animation of the movement of all nodes while they move towards an objective. The simulator generates a data file describing the network changing topologies needed by the HGA module.

- MANET traffic generator - creates random connections between nodes, according to scenario-imposed restrictions. Traffic model is implemented in such a way that the assigned leader of each group is responsible for forwarding orders or information to other group leaders. The HGA module also uses these data to establish links between nodes.

- Hybrid Genetic Algorithm (HGA) - At each time frame, a hybrid genetic algorithm with reparation of individuals and heuristic crossover and mutation operators finds efficient routes that preserve maximum energy availability at network level, reducing the probability of communications disruption. It also computes shortest path routes for the sake of comparison.

\subsection{MANET Simulator}

Figure 4 shows the MANET simulator with a view of the planned deployment of the operational groups and snapshots of the area of operation at the start and at the end of simulation time. 


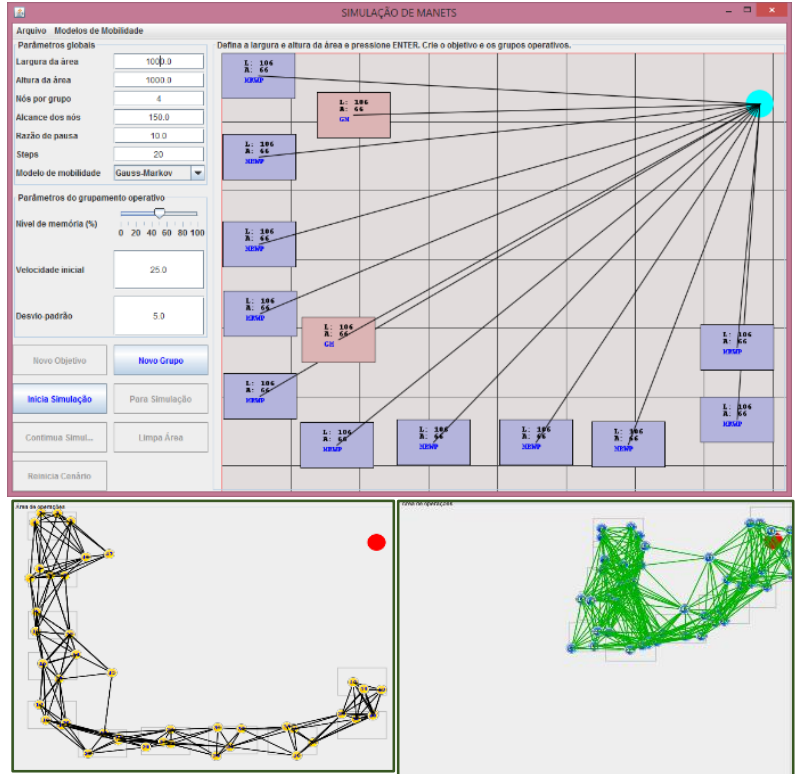

Figure 4 - Deployment, initial and final locations

The MANET Simulator offers a graphical animation of a moving MANET, generated from a set of adjustable parameters related to the geographical and operational mission configuration. In addition, it generates a data file containing brief descriptions of the network, some mission related data and a sequence of adjacency matrices, one for each step of the simulation. The simulation may be interrupted and re-initiated, and scenarios may be saved or loaded from disk and modified. Some of the simulation parameters are listed below:

1.The number of time steps.

2.The dimensions of the rectangular area of operations

3.The position and the dimensions of mission's objective.

4.For each group:

a. The number of elements (nodes).

b. The range of their devices.

c. The probability $p_{\mathrm{s}}$ of pause between movements.

d. The location and the dimensions of a rectangular area were the elements are initially located, according to a choice of probabilistic models (bivariate uniform or bivariate Normal).

e. The mobility model (and related parameters) for each group. If a node reaches the boundary of the area of operation or the limits of its group area (for group models), it bounces back according to some reflection rule.

\subsection{Mobility Models}

Many researchers use static or random mobility models to evaluate routing protocols in ad hoc networks [9] [10] [11]. According to these models, the speed and direction of motion in a new time interval are not correlated to their past values in the previous epoch and may show unrealistically sudden or brisk changes. In addition, they do not consider common objectives. Some studies [12] [13] [14] question the validity of the results when these models are used. To overcome these difficulties, military experts (see, for example [15] and [16]) were consulted and modifications to known models were introduced, in order to account for the necessary realism of the type of missions considered here.

The MANET simulator permits an on-the-run selection of geographical and mobility parameters, in order to simplify the process of matching the experts view of the planning problem.
Some of the mobility models so far implemented are the following:

\section{a. Random Walk (RW)}

Einstein originally described the Random Walk process to emulate the unpredictable movement of particles in 1905 [17], to model the Brownian Motion. This model may be used in situations when nodes move independently and unpredictably. It is a simple, albeit unrealistically, model when human movements are present. At each time interval $t$, each node changes its direction $\theta(t)$ sampling from a Uniform distribution ranging from $(0,2 \pi]$. In a similar way, the new speed follows a Uniform $\left(0, \mathrm{~V}_{\mathrm{Max}}\right)$ distribution, or a Normal $\left(\mu_{\mathrm{v}}, \sigma\right)$ distribution. Therefore, during time interval $t$ a node moves with the velocity vector $\vec{v}(t)=[v(t) \cos \theta(t), v(t)$ $\sin \theta(t)]$.

\section{b. Gauss-Markov (GM)}

The Gauss-Markov mobility model introduces a tuning parameter that allows the adjustment of the degree of randomness of the movement and it has been widely used [14] [18] to model ad hoc networks. Each node moves with a parametrized velocity vector

$\vec{v}(t)=\vec{\alpha} \cdot \vec{v}(t-1)+(1-\vec{\alpha}) \cdot \vec{v}+\vec{\sigma} \cdot \sqrt{1-\vec{\alpha}^{2}} \cdot \vec{W}(t-1)$, where $\vec{v}(t)=\left[v_{x}(t), v_{y}(t)\right], \quad \vec{W}(t)=\left[W_{x}(t), W_{y}(t)\right]$ is an uncorrelated random Gaussian process with mean zero and variance $\sigma^{2}, \vec{\alpha}=\left[\propto_{x}, \propto_{y}\right]$ is the memory level, and $\vec{v}=$ $\left[v_{x}, v_{y}\right]$ and $\vec{\sigma}=\left[\sigma_{x}, \sigma_{y}\right]$ represent the asymptotic (as $t \rightarrow \infty$ ) vector mean and vector standard deviation, respectively. At each time interval, $\vec{v}(t)$ is updated according to the following two-dimensional field:

$$
\left\{\begin{array}{l}
v_{x}(t)=\alpha v_{x}(\mathrm{t}-1)+(1-\alpha) v_{x}+\sigma_{x} \sqrt{1-\alpha^{2}} w_{x}(t-1) \\
v_{y}(t)=\alpha v_{y}(\mathrm{t}-1)+(1-\alpha) v_{y}+\sigma_{y} \sqrt{1-\alpha^{2}} w_{y}(t-1)
\end{array}\right.
$$

Liang and Haas [19], who proposed the model, claim it is possible to model different mobility scenarios using equations (1), since time-dependency may be easily adjusted by the memory level parameter $\propto$. For example, if $\alpha=0$, the model is memoryless, i.e., the velocity of a node at time $t$ is a function only of the fixed drift velocity $\vec{v}$ and the Normal random variable $\vec{W}(t)$. This is precisely the Random Walk model. On the opposite side of the spectrum, if $\alpha=1$, meaning a complete memory effect, $v_{x}(t)=v_{x}(t-1)$ and $v_{y}(t)=$ $v_{y}(t-1)$, i.e., the movement is linear. If $0<\alpha<1$, the current velocity is a parametrized function of both its previous velocity and the Normal random variable.

\section{c. Mixed Random Waypoint (MRWP)}

To evaluate the impact of real-life scenarios in the performance of routing protocols in ad hoc networks, the Mixed Random Waypoint was developed [2]. In this model, there are no sudden or brisk changes of direction, allowing for group movements with uniformly distributed random speed. Uniformly distributed random pause times are also part of this model, providing realism and generalization.

In this model, each node moves from a pre-assigned random origin, following either a bivariate uniform distribution (occupying a small area) or a bivariate Normal distribution (concentrated around the group leader) to a randomly selected destination constrained to be in the interior of a sub-area chosen inside the overall simulation area. Then, all groups move in the same general direction, but they will reach distinct points, restricted within the limits of the area. Two kinds of movement in this scenario are considered: the movement of individual nodes with respect to the center of its group; and the joint group movement, i.e., the movement of the group center. As a basis to the model, the Random 
Waypoint Model [5] and the Mixed Waypoint Model are employed, respectively. The Random Waypoint model divides the complete path of a Mobile Node (MN) in periods of movement and periods of pause. Initially, a MN stays at a fixed point for a certain interval of time and then it moves to a chosen location with a uniform random speed. The Mixed Waypoint model is an a variation of the Reference Point Group Mobility (RPGM) model [18], and it represents the random joint movement of a group of $\mathrm{MN}$ as well as the individual movement of nodes inside each group. Group movement is based on the trajectory of a logical reference point (center) of the group. This reference point is used to compute the random movement of each MN. All the MNs in a group have only one reference point, so that the integrity of the joint group movement towards the common objective is preserved. Each MN forming groups move with a uniformly distributed speed with a given mean value and a pause time also uniformly distributed.

\subsection{Traffic Generator}

As mentioned, the traffic generator creates a file containing data associated to random connections between nodes, according to scenario-imposed characteristics, such as the number of nodes, the number of connections and the mission duration. Connections are initiated at random times, which are uniformly distributed over the simulation period. The generated traffic pattern shows hierarchical/functional characteristics, seeking to model military or rescue operations procedures.

\subsection{Hybrid Genetic Algorithm (HGA)}

The particular MANET routing problem needs to solve six specific issues, before one can efficiently employ the classical methods of Genetic Algorithms [20]:

1. The efficiency and effectiveness of chromosome coding, with respect to the representation of valid routes;

2. An efficient methodology to generate the initial population;

3. A fitness function that privileges the dynamic performance of the network;

4. Crossover an mutation operators that preserve the feasibility of individuals;

5. Appropriate GA parameters (population size, crossover and mutation probabilities, elitism and immigration taxes, etc.);

6. MANET routing is clearly a combinatorial optimization problem, with a relatively simple repair strategy. One alternative may be the use of penalty functions for unfeasible individuals. However, the search space is severely non-convex and discontinuous. Furthermore, with high probability, it has many and large unfeasible regions, implying the non-convergence of those functions.

\section{Chromosome Representation}

The choice of genotypes must consider that, in classical binary representation, genetic material interchange among parents frequently occurs in cut points located inside the code for integer values. This can generate a completely new set of phenotypes, failing to propagate the genetic heritage from parents to children. Let's consider a MANET with $N$ nodes. Each chromosome is a variable-length sequence of different positive integers $g_{i} \in\{1,2, \ldots, n\}, 1 \leq i \leq M$, where each gene $g_{i}$ identifies a single node that is part of a non-cyclic route from a designated origin $o$ to a destination node $d$. $M \leq N$ is the number of nodes in the route $\pi$. The locus of each gene represents the order of the corresponding node in the route. The first and last genes represent, respectively, the nodes $o$ and $d$.

\subsection{HGA Description \\ For each time period $t$ do:}

1) Check if a direct route exists from origin node $o$ to destination node $d$, i.e., if $d \in N b(o)$, the neighborhood of $o$.

Otherwise,

2) Use Depth First Search (dfs) to verify that $o$ connects to d. If they are connected:

a) Initialization $-1^{\text {st }}$ generation, $g_{1}$ (generate $\mu$ feasible individuals)

(1) Start the population with the route found in step 2).

(2) Generate new individuals: randomly select a node $n \in N b(o)$ and set nodes $o$ and $n$ as visited.

(a) Repeat step (2) above, now from node $n$, skipping visited nodes (as in a probabilistic-like dfs).

(b) If process fails (a newly found node $k$ has only visited nodes in his neighborhood), go back to step (2).

(3) A feasible chromosome $C$ is generated when node $d$ is found. This algorithm converges with probability 1 , since there is at least one route from $o$ to $d$ (already found in step (1)).

b) Fitness Evaluation

(1) SP - a measure similar to shortest path computation is included in order to establish a benchmark to compare to other fitness evaluation measures. The cost to be minimized in route $\pi$ is $\mathrm{C}(\pi)=$ $\sum_{\mathrm{i} \in \pi} \mathrm{C}_{\mathrm{i}}$, where $\mathrm{C}_{\mathrm{i}}$ is simply the energy consumption at each node belonging to the route.

(2) EA - Energy-aware fitness function

Minimize the total cost of route $\pi$ is $\mathrm{C}(\pi)=$ $\sum_{\mathrm{i} \in \pi} \mathrm{C}_{\mathrm{i}}, \mathrm{C}_{\mathrm{i}}=\left(\tau_{\mathrm{i}}+\rho_{i}\right)\left(\frac{\mathrm{F}_{\mathrm{i}}}{E \mathrm{E}_{\mathrm{i}}}\right)^{\alpha}$, where

$\tau_{\mathrm{i}}=$ transmission energy consumption of node $i$

$\rho_{i}=$ reception energy consumption of node $i$

$\mathrm{F}_{\mathrm{i}}=$ initial battery capacity of node $i$

$\mathrm{ER}_{\mathrm{i}}=$ remaining energy level at node $I$ after message routing

$\alpha=$ low-energy weight factor

(3) MM - Maximize minimum battery charge in route $\pi$, i.e., $\mathrm{C}(\pi)=\min _{i} E R_{i}$

c) Elitism

In order to keep the best individuals of a generation, protecting them from changes due to crossover and/or mutation, they are transmitted unchanged to the next generation. Consequently, the $\lambda_{e}$ best individuals are included in the next generation.

d) Selection

The selection procedure used by the algorithm is rank or roulette wheel.

e) Heuristic crossover

1. 1-point crossover - parents must have at least one common gene (node) in the route from $o$ to $d$. Select the first of these nodes and switch the remaining sub-routes in children. If there are no common genes, don't perform the crossover.

2. If the result of the crossover generates cycles, use the following repair algorithm:

a) Get the first cyclic node. 
b) Remove the sub-route between this node and the first time it reappears in the route.

c) Recursively return to step a) until there are no more cycles.

f) Mutation

1. Randomly select one node from the route and, using this node as a surrogate origin, generate a new feasible random sub-route to the destination node $d$.

2. After mutation, proceed with next step, route optimization.

g) Route optimization

If applicable, for all new route $\pi$, shorten the route (from $g_{2}$ inclusive), checking at each node $k \in \pi$ if $d \in N b(k)$.

h) Immigration to enhance diversity

$\lambda_{m}$ immigrants are included in the intermediary population $\left(\mu+\lambda_{e}+\lambda_{m}\right)$. Discard the $\left(\lambda_{e}+\lambda_{m}\right)$ worse (with respect to fitness) individuals.

\section{3) Stopping criteria}

a) Reach the maximum number of generations, $g_{\max }$, or

b) The fitness of the best individual is unchanged for $m$ generations.

Update the topology of the network, moving nodes according to their mobility models. Remove devices with depleted batteries, if any, and go back to step 1) for a new route (connection)

\section{SIMULATION AND RESULTS}

\subsection{Scenarios}

This section presents a selection of simulations used to evaluate the performance of the new routing model under different parametrization strategies, searching routes that prioritize network energy efficiency in a MANET environment. Furthermore, estimates of the minimum number of active devices required to ensure network connectivity are computed, considering the unavoidable energy discharge on some nodes. Realistic military or search-and-rescue operations in a single objective, limited area are considered, trying to achieve some degree of generalization. These operations require a high degree of coordination among groups and there is no interaction with the object of search, due, possibly, to the precarious conditions of the people being searched. Three different scenarios (with 23, 35 and 47 nodes) were designed, in order to find the best network configuration, emphasizing parameters selection procedure. These scenarios are described below:

- Scenario 1 - five groups of four elements each, two components in an advanced position, one controller (leader), adding up to 23 ad hoc nodes.

- $\quad$ Scenario 2 - eight groups of four elements each, two components in an advanced position, one controller, adding up to 35 nodes.

- Scenario 3 - eleven groups of four elements each, two components in an advanced position, one controller, adding up to 47 nodes.

For example, one of the scenarios (Figure 4 above) represents a search party consisting of 47 people of different specialties, including health professionals, volunteers, etc., looking for victims at or near the objective. Each person is carrying a personal communicator capable of joining an ad hoc network. The team is divided into 11 groups, each one with four components; in this example scenario, there are two observers positioned in an advanced location with respect to the other groups to keep them informed about any important developments. It is also simple to place observers anywhere in the area of operation.

Groups have a common objective, and collaborate with each other. Traffic pattern consists of orders, information and other mission-related data from group leaders to other groups and to a control unit, which is located in one of the groups. The scenario allows for a high probability of partitioning of the network, due to the mobility of the nodes, continually changing the connectivity situation among them. A realistic situation is approximately obtained by choosing carefully how to model these movements and by appropriately selecting traffic parameters.

The algorithm performance is evaluated with respect to the three studied measures of effectiveness, or fitness functions, used in the HGA (see Table 1). By extending the simulation time or increasing traffic density, it is possible to reach a fair estimate of the minimum battery inventory level to accomplish the mission.

Table 1 - Parameters for the Simulation and the HGA

\begin{tabular}{|l|l|l|l|c|}
\hline \multirow{3}{*}{ Nodes } & Scenario 1 & 23 & Population & 60 \\
\cline { 2 - 5 } & Scenario 2 & 35 & Crossover & 0.8 \\
\cline { 2 - 5 } Simulation area & Scenario 3 & 47 & Mutation & 0.2 \\
\hline Comm. range & $800 \times 600$ & & Max Generations & 20 \\
\hline Average speed & 150 & Selection & Rank \\
\hline Mobility models & MRWP e GM & Immigration & 5 \\
\hline \# of connections & 20 & Initial Energy & 30 \\
\hline Max. pause rate & $10 \%$ & & \\
\hline
\end{tabular}

The MANET simulator generated the before mentioned scenarios, based on expert counselling, which helped to determine the appropriate parameters to emulate a real-life situation. Actually, a new Mixed Waypoint mobility model [2] is implemented to follow these specifications, with its parameters also listed in Table 1 to model the movement of the nodes in these simulations. The traffic generator produced twenty random connections between pairs of nodes, following the restriction that only group leaders should communicate with other group leaders. The remaining nodes became routers, providing itineraries for the communications. There is, initially, at least one route for each traffic demanded, such that any failure to find an appropriate route would be credited to the HGA's inability to find a route, or to the partition of the network, caused by previous energy consumption in any of the required nodes (transmitter, router or receptor).

\subsection{Measures of Effectiveness}

Transmission and reception energy consumption is set to 4 and 3 units, respectively. A router node, therefore, consumes 7 units. To stress the network, a low initial energy level is set for all nodes. For each connection demand generated by the Traffic Generator, the HGA uses the network connectivity pattern, updated by the MANET Simulator for that particular instant $t$, and finds the optimal route. This route data is then used to update the energy levels of all nodes. This process continues until the end of the simulation time. For each scenario, all three fitness functions are used to compute optimal routes for each connection. For each scenario and for each fitness function (EA, SP and MM), the HGA runs 50 times, changing the seeds of the random number generators. A set of measures are computed from the average results of these runs. Table 2 summarizes the results found for the following measures: 
- Network Energy Capacity - total (all nodes) remaining battery charge at the end of the simulation.

- Failure Rate - percentage of messages that could not be forwarded due to network segmentation or disruption.

- Consumed Routers - percentage number of nodes selected for routing service with insufficient battery for this task, causing a network partitioning.

- Consumed Nodes - percentage number of nodes with battery level below the minimum threshold, i.e., unable to transmit or receive messages.

- Minimum Charge - minimum charge of any node in the network at the end of the simulation.

\subsection{Results and Analysis}

Table 2 summarizes the results. For scenario 1, it may be observed that fitness functions EA and MM show the same result, with fewer depleted nodes at the end of the simulation, and providing slightly smaller failure rates than shortest-path routes (SP). In any case, however, approximately $20 \%$ of the connections could not be made due to network partition caused by node exhaustion, as indicated by the Consumed Routers measure. It may readily concluded that, for scenarios with a small number of nodes, the fact that alternative routes are rare or do not exist imposes a fast exhaustion rate that may jeopardize operations where communications reliability is vital. Accordingly, the analytical environment may be employed in the planning phase of this kind of mission to assess the need for supplies or to dimension the proper number of stations to guarantee, to a certain level of reliability, the success of the operation.

In scenario 2, an improvement in Failure Rate for all methods is observed. EA and MM show similar results, with EA being slightly better. EA allows for $100 \%$ of connections, keeps the nodes with a better energy state, with less consumed nodes and routers at the end of the simulation. EA provides the largest life span for the network. SP presents difficulties to balance energy consumption at network level, selecting always the shortest route, with no concern about energy conservation. The same node is used a number of times in different connections, increasing the probability of node exhaustion and network partitions.

In scenario 3, the EA criterion shows a very good result, with completion of all demanded connections and a good energy balance. At the end of the simulation, minimum charge is maximized and the number of exhausted nodes and routers is minimized. This fitness function prioritizes routes containing nodes with greater energy capacity, accepting longer routes (observe the smaller value for Energy Capacity) to preserve energy at system level. At the end of the simulation, even though $6 \%$ of the nodes are excluded from the available routers list, only $1 \%$ of them are actually eliminated from the network (but notice that, on average, the minimum charge for EA is superior to the energy required for transmission. MM and SP exhaust more nodes and show smaller system balance capability, as the measures Consumed Routers and Consumed Nodes indicate. For this scenario, SP reaches the largest value for Energy Capacity at the end of the simulation, because it always selects routes with fewer nodes in it. The downside is that fewer routes are found, since some nodes are exhausted and the network becomes partitioned.

In scenarios like the ones being evaluated, where groups cooperate to fulfil a common task, the exhaustion of any node may cause the partition of the whole network, isolating groups. This can be disastrous for the accomplishment of the mission. Consider a search-and-rescue operation looking for survivals of a plane crash in a hostile or difficult environment. When one group localizes the survivors, it must transmit its position and maybe some other data to the medical group. Then, a feedback communication will exist, perhaps some information concerning first-aid procedures. If the network is partitioned and these groups can't communicate, the mission fails.

Table 2 - - HGA results for the three fitness functions

\begin{tabular}{|c|c|c|c|c|c|c|c|c|c|}
\hline & \multicolumn{3}{|c|}{ Scenario 1} & \multicolumn{3}{|c|}{ Scenario 2} & \multicolumn{3}{|c|}{ Scenario 3} \\
\hline & EA & SP & $\mathrm{MM}$ & EA & SP & MM & EA & SP & MM \\
\hline Energy & 382 & 403 & 382 & 516 & 585 & 536 & 764 & 861 & 808 \\
\hline Failure Rate \% & 20 & 28 & 20 & 0 & 11 & 3 & 0 & 5 & 3 \\
\hline Cons. Routers & 17 & 21 & 17 & 10 & 19 & 10 & 6 & 15 & 9 \\
\hline Cons. Nodes \% & 9 & 11 & 9 & 1 & 16 & 4 & 1 & 11 & 4 \\
\hline Min. Charge & 0 & 0.5 & 0 & 3 & 0 & 1 & 4.25 & 1.25 & 2 \\
\hline
\end{tabular}

A somewhat less critical situation was also simulated, when nodes start with an initial capacity of 40 . Table 3 shows the average results. With this less stringent scenario, EA and MM achieve the same result, but once more, the SP policy inflicts an important penalty in terms of consumed nodes ( $9 \%$ of the nodes become unavailable as routers).

Table 3 - Initial capacity increased to 40

\begin{tabular}{|c|c|c|c|}
\hline \multicolumn{1}{c|}{} & EA & SP & MM \\
\hline Energy Capacity & 875 & 910 & 875 \\
Failure Rate & $0 \%$ & $0 \%$ & $0 \%$ \\
Consumed Routers & $0 \%$ & $9 \%$ & $0 \%$ \\
Consumed Nodes & 11 & 1 & 11 \\
\hline
\end{tabular}

Figure 5 shows the failure rate and average energy consumption for each combination of the predefined scenarios with the route-finding fitness functions.

The network becomes feasible with 35 nodes and the EA optimization policy. For a network with fewer nodes, the MM method behaved similarly to EA. With more stations, however, there are more alternative routes to forward a message and EA is able to find routes that are more efficient, preserving system (network) availability for a longer period at the expense of a larger average consumption of energy.
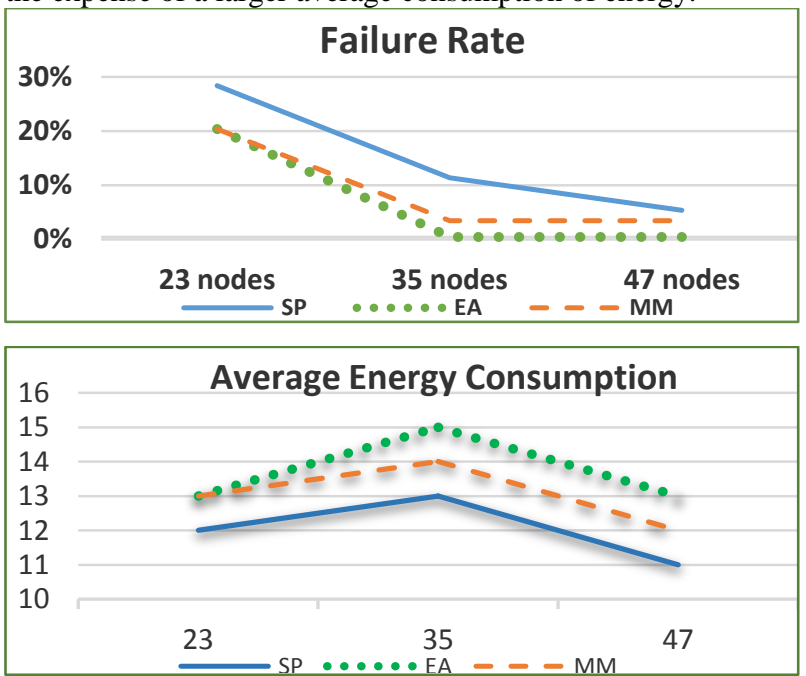

Figure 5 - Comparing policies 
Figure 6 presents a comparative view of the survival rate (the percentage of active nodes in the network) versus time (measured in simulation steps) for the three scenarios.
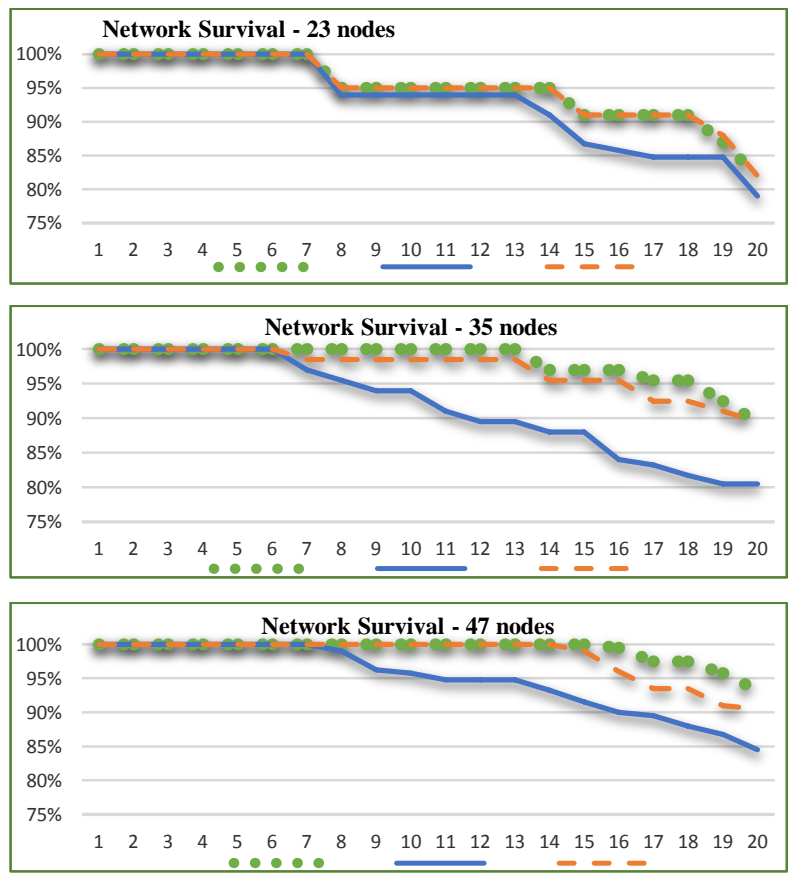

Figure 6 - Survival rate for different policies

It may be noticed that, initially, all methods have the same performance, since all nodes start with the same energy level, and shorter routes are favored. As time passes, they consume energy, and the route-finding policies starts to behave differently. SP performs poorly in all situations, while EA outperforms (or, at least, matches) all other methods, providing superior network survival. The results of the simulations are conservative, in the sense that traditional routing protocols require more energy consumption, due to the numerous route discovery processes caused by the frequent partition of the network - here nodes consume energy only when forwarding messages.

\section{CONCLUSIONS}

In this work, a new model is developed to aid the planning and analysis of communications-intensive Mobile Ad Hoc Networks (MANET), with respect to the allocation of energycritical communication equipment in realistic missions such as military or search-and-rescue operations, usually occurring in inhospitable environments. A graphical simulation tool and a hybrid genetic algorithm (HGA) with reparation of individuals and heuristic crossover and mutation operators are designed to provide the means to reach an efficient routing policy, attempting to maximize the preservation of energy availability at network level, reducing the probability of communications disruption. The system is flexible and customizable, providing a means to help mission planning, including the provision of adequate power supply for the large number of devices typically found in such MANETs.

The simulator developed for the evaluation of the proposed algorithm implements mobility models chosen according to field operations experts, in order to lend realism to the whole process. The simulator provides input data for the HGA. One new model, Mixed Random Waypoint (MRWP), is introduced to evaluate the impact of real-life scenarios in the performance of routing protocols in ad hoc networks. The simulator allows for the customization of a variety of scenarios, the establishment of different operational-driven supply policies and the estimation of the probability of mission success.

To forward a message, some kind of shortest path technique is usually adopted in conventional routing protocols for ad hoc networks, seeking routes with a minimum number of hops. The results indicate that these are not necessarily the best solutions, when energy conservation issues are important.

The algorithm is designed to extend the lifetime of an ad hoc network, avoiding, when possible, routes containing nodes with a low energy level. In addition, it incorporates a systemwide fitness function. In the analysis, three types of optimization methods in search of routes (Shortest Path - SP; maxmin - MM; and the proposed fitness function Energy Aware - EA) were compared, using scenarios with different numbers of devices (23, 35 e 47 nodes). The results indicate that, for smaller networks (with fewer devices), the methods MM and EA show a similar behavior, being both better than the standard SP. However, when the number of devices increases, the fact that EA generates more alternative routes from a source to a destination tends to reach solutions that are more efficient. By balancing the whole network energy consumption, EA prolongs the network lifetime, avoiding partitions in the MANET.

Of course, lengthier routes will increase not only the waiting time for route selection but also the time delay for forwarding packets. On the other hand, fewer inefficient routing rediscovery processes are necessary, since the probability of network partition is minimized. The before mentioned processes, which are common in conventional MANET protocols, are time and energy intensive. As the number of nodes increase in the network, the experiments (with 11 groups and 47 nodes) indicate that EA provides the best results.

In summary, in this paper, this paper describes how a hybrid genetic algorithm associated with a simulation tool and proper mobility models may be used to optimize energy-aware ad hoc wireless networks. The proposed methodology prioritizes energy-wide system availability and reduces the number of network partitions, extending its lifetime and providing a better probability of mission success.

\section{REFERENCES}

[1] Z. Haider and F. Shabbir, "Genetic Based Approach for Optimized Routing in Maritime Tactical MANETs," in Proceedings of 11th International Bhurban Conference on Applied Sciences \& Technology - IBCAST, Islamabad, Pakistan, 2014.

[2] I. C. M. Pereira, Análise do Roteamento em Redes Móveis Ad Hoc em Cenários de Operações Militares, UFRJ, Rio de Janeiro, 2004.

[3] C. E. Perkins, E. M. Belding-Royer and S. R. Das, "Ad Hoc On-Demand Distance Vector (AODV) Routing," November 2002. [Online]. http://www.ietf.org/internetdrafts/draft-ietf-manet-aodv-12.txt.

[4] C. Perkins and P. Bhagwat, "Highly dynamic destination-sequenced distance vector routing (DSDV) for mobile computers," in ACM SIGCOMM Conference on Communications Architecture, Protocols and Applications, 1994. 
[5] D. B. Johnson and D. A. Maltz, "The dynamic source routing protocol for mobile ad hoc networks," Intenet Draft, draft-ietf-manet-dsr-06.txt, 2002.

[6] V. Park and S. Corson, "A Highly Adaptive Distributed Routing Algorithm for Mobilie Wireless Networks," in INFOCOM, 1997.

[7] Z. Huang, R. Yamamoto and Y. Tanaka, "A Multipath Energy-Efficient Probability Routing Protocol in Ad Hoc Networks," in IEEE International Conference on Advanced Communications Tecnology, Seul, 2014.

[8] Z. Michalewicz, Genetic Algorithms + Data Structures = Evolution Programs, New York: Springer, 1996.

[9] D. S. Kumar and V. B. Kumar, "Energy-aware Multicast Routing in MANETs based on Genetic Algorithms," in 16th IEEE International Conference on Networks (ICON’08), New Delhi, India, 2008.

[10] J. Zhao, X. Jiang and J. Sha, "A Study of the Relationship between Mobility Model of Ad Hoc Network and its Connectivity," Journal of Computers, vol. 9, no. 4, April 2014.

[11] J. Abdullah, "Multiobjectives GA-based QOS Routing Protocol for MANET," International Journal of Grid and Distributed Computing, vol. 3, no. 4, Dec. 2010.

[12] T. Camp, J. Boleng and V. Davies, "A Survey of Mobility Models for Ad Hoc Network Research," Wireless Communication and Mobile Computing (WCMC): Special issue on Mobile Ad Hoc Networking: Research, Trends and Applications, vol. 2, no. 5, pp. 483-502, 2002.

[13] F. Fitzek, F. H. P. Fitzek, T. K. Madsen, R. Prasad and M. Katz, "Impact of Node Mobility on the Protocol Design of Selforganizing Networks," in Proceedings of
Wireless World Research Forum, 2004.

[14] A. Jardosh, E. M. Belding-Royer, K. C. Almeroth and S. E Suri, "Towards Realistic Mobility Models for MANET," in MobiCom'03, San Diego, CA, 2003.

[15] D. V. Campos and R. B. Seixas, "Command and Control a Low Cost Framework to Remotely Monitor Military Training," in Proceedingds of Spring Simulation Multiconference in Military Modeling and Simulation Symposium, Boston, 2011.

[16] D. V. Campos, Sistema de Jogos Didáticos: uma Abordagem Prática de Simulação Construtiva a Serviço dos Jogos de Guerra do Corpo de Fuzileiros Navais, Rio de Janeiro: Escola de Guerra Naval, 2015.

[17] S. Brush, "A history of random processes: Brownian movement from Brown to Perrin Arch," History of Exact Science, vol. 5, pp. 1-36, 1968.

[18] X. Hong, T. J. Kwon, M. Gerla and D. Lihu, "A Mobility Framework for Ad Hoc Wireless networks," in MDM'01: Proceedings of the Second International Conference on Mobile Data Management, 2001.

[19] B. Liang and Z. Haas, "Predictive distance-based mobility management for PCS networks," in Proceedings of the Joint Conference of the IEEE Computer and Communications Societies (INFOCOM), New York, 1999.

[20] J. H. Holland, Adaptation in natural and artificial systems: an introductory analysis with applications to biology, control and artificial intelligence, Ann Arbor, USA: University of Michigan Press, 1975. 